\title{
Disclosure of exact protocols of fermentation, identity of microorganisms within consortia, formation of advanced consortia with microbe-based products
}

\author{
Yoav Bashan 1,2,3 . S.R. Prabhu ${ }^{4}$ - Luz E. de-Bashan ${ }^{1,2,3} \cdot$ Joseph W. Kloepper ${ }^{3}$ \\ Published online: 8 May 2020 \\ (C) Springer-Verlag GmbH Germany, part of Springer Nature 2020
}

In the last 40 years, the concept of microorganisms that can enhance plant productivity matured into a research field, stretching from in vitro experiments, greenhouse and field trials done for research purposes, to commercial products applied worldwide. Currently numerous companies, big and small, are producing and marketing a large variety of seed inoculants and other microbial products for agricultural and horticultural uses (Bashan et al. 2014; Calvo et al. 2014). The market value of plant growth-promoting rhizobacteria/ bacteria (PGPR/PGPB), biological control agents, and a variety of biostimulants was valued at USD 6.00 Billion in 2016 and is projected to reach 14.65 Billion by 2023 , growing at a compound annual growth rate of $13.80 \%$ from 2017 (https:// www.marketsandmarkets.com/Market-Reports/top-10-trendagricultural-biological-market-139215554.html).

The scientific community engaging in research with these microorganisms is interested mainly in new developments, new products and new scientific concepts. As such, precise validation or simple confirmation of previous results or repetition of already published studies has very low priority. Because every scientific journal is interested in innovation, these results are seldom, or never, published. The direct user

Luz E. de-Bashan

luz@bashanfoundation.org

$\triangle$ Joseph W. Kloepper

kloepjw@auburn.edu

1 The Bashan Institute of Science, 1730 Post Oak Ct, Auburn, AL 36830, USA

2 Environmental Microbiology Group, Northwestern Center for Biological Research (CIBNOR), Av. IPN 195, 23096 La Paz, B.C.S, Mexico

3 Department of Entomology and Plant Pathology, Auburn University, 301 Funchess Hall, Auburn, AL 36849, USA

4 TerraBioGen Technologies, 8536 Baxter Place, Burnaby, B.C V5A 4T8, Canada of such published information is the inoculant industry. Naturally because of intellectual property, issued information of failed experiments is mostly unavailable. Yet, quite a few studies repeated on commercial inoculants from various industries did not provide the benefits expected from these products (Denton et al. 2002; De Bruin et al. 2010; Karamanos et al. 2010). Such failures are better known in the biomedical industry (Petsko 2010).

We identified four potential pitfalls responsible for such failures in the PGPR/PGPB research field; (i) improper description of fermentation processes by the original publication; (ii) improper description of consortia when used, (iii) improper description how consortia are made of, (iv) most times, the publications also don't clearly disclose formulation techniques, exact dosage of microbial products used per unit area, soil physico-chemicals conditions etc., which could seriously impact the performance of microbial products, and (v) very few formal publications describing failure of a set of experiments (Bacilio et al. 2017; Karamanos et al. 2010; Martínez-Viveros et al. 2010).

All PGPR/PGPB inoculants are culturable bacteria. Consequently, proper fermentation to produce large population of bacteria to be later formulated into an inoculant is one of the most essential features of inoculant production (Bashan et al. 2014; Calvo et al. 2014). Most publications followed the first original one on any PGPR/PGPB plant interaction and sometimes even in the first publication, to save printing space in the journal, the fermentation process is described briefly or as a reference from another PGPR/PGPB system following a statement "with small modifications" where the changes are described, but rarely a fully detailed protocol is provided. This first paper is commonly used as a reference for future publications on specific PGPR/PGPB interaction.

While it is common that the experimental laboratory uses analytical ingredients for small fermentation studies, to save costs, the industrial manufacturers of inoculants use less purified ingredients. From numerous microbiological studies it is 
well established that addition of impurities into culture medium may significantly change the outcome of the fermentation for formation of other metabolites responsible for plant growth promotion or creating plant growth inhibition. Additionally, small technical details may also play a factor which makes repetition of the original fermentation a challenge for the industry. These parameters could involve difference in stirring, formation of biofilm, fluctuation in temperature, oxygen gradient within the fermenter, volume of the batch fermentation, harvesting time and harvesting procedures. These small but sometimes crucial technical details are seldom published because they lack "sufficient novelty for our broad readers" as most journals put it to the authors who submit such technical papers.

It is commonly understood that competition between journals for the most novel articles and ideas will not change as this directly affected their metrics and in printed journal will also significantly increase the size of Materials and Methods section for the benefit of only one client of these journals, the industry. A practical solution can be suggested. Almost all important journals contain a section of "supplementary material" available to all readers or subscribers. This section provides ample space to include detail data, mostly for statistical purposes or complicated mathematical/bioinformatical analyses. Addition of detailed fermentation protocol will make no difference to the space allocated to the publication and will satisfy the necessities of scientists who need to repeat the experiment.

In earlier period of the PGPR/PGPB research field a specific species or strain was used and is even true today in many experimental and commercial Azospirillum spp. and Bacillus spp. based inoculants. Verification and monitoring of a single species, once a proper molecular procedure is established, is fairly easy and reliable (Posada et al. 2016; Trejo et al. 2012). Over the last decade, combination of bacterial species, specific strains, and mixture with other beneficial microbes, consortium, is being tested and formulated as inoculants. The issue of proper identification of each species and strain within such inoculant was addressed in a previous editorial (Bashan et al. 2016). Yet, when consortium formation is addressed, more technical questions need to be additionally raised to the proper identification of the members of such consortia. Two types of consortia are known, a simple one and a complex one. Simple consortium can consist of simply mixing PGPR/PGPB/AM fungi fermented separately before inoculation. Consortia can be formed by fermenting more than one PGPR/PGPB together in a medium suitable for both. The more species/strains in the fermentation process the more complex the outcomes, because species can interact and affect each other growth and production of PGP metabolites. Once such consortia are formed, a detailed analysis of the population is essential to know and also the quantity of PGP metabolites formed. This information can be included in a table in Materials and
Methods together with the identification of the members of the consortium.

Complex consortia are by far more difficult to handle regarding PGPR/PGPB. In addition to the limitation presented by a simple consortium, there are additional complications inherit from time and source of the consortium. There are two types, consortia formed over time such as in the food industry, and unknown consortia formed which cannot be defined due to insufficient knowledge at this time. Consortia can be formed as a starter of an industrial product such as diary fermentation, wine, tequila and other alcoholic fermentations where the main organism is known but not the supportive microorganisms that were added experimentally or accidentally over long period of time. These microorganisms are typically regarded as industrial secrets. In the case of PGPR/PGPB, if the developer is reluctant to disclose them, there is no scientific value of publishing the paper. Once the information is lost, this consortium is lost forever.

The second complex consortia are the ones planed for the future based of microbiomes/metagenomics analyses. Currently, studies on metagenomics and microbiome of certain plant organs are highly descriptive considering the plant and its associated microorganisms as metaorganisms (Berg et al. 2016). Depending on the quality and type of the molecular analysis, it typically yields a long list of microorganisms with high speculative functional description depend on similarities of genes and relatedness among species (Nannipieri et al. 2019; Schöler et al. 2017; Vestergaard et al. 2017). The relation to PGPR/PGPB are only indirectly established because the plants function well and sometimes growth promotion occurred but it is unclear who caused what effect in the metaorganism (Bakker et al. 2013). In times when this descriptive evaluations are predicted to be transformed from experiments done in vitro in petri dishes with miniature model plants such as Arabidopsis to more functional microbiome capable of producing potential data for practical use (Berg et al. 2013), this will create, by default, a complex consortium mainly because of lack of knowledge about specific interplay within the plant holobiont that is yet to be explored. When this occurs, the only solution is to give full description of the microorganisms involved together with the identified PGPR/PGPB as a description of a consortium where all the functions of all microbial members and their interaction are unknown.

Any reading through extensive PGPR/PGPB literature shows uninterrupted continuous success of the approach with hardly any failure reported. In reality, many studies failed, but this failure is not represented in the literature which gives a misleading view of the state of art of the field. Only rarely failures of experiments were formally described (See above references). The opinion of most journals on novelty publications notwithstanding, there is a need especially for industrial scientists to know which approaches failed to avoid perusing dead-end causes. 
In summary, we propose that new manuscripts that do not contain the above information on fermentation of microorganisms or proper description of a consortium that was use should not be considered for publication. Simultaneously, publication of negative results is encouraged.

\section{References}

Bacilio M, Moreno M, Lopez-Aguilar DR, Bashan Y (2017) Scaling from the growth chamber to the greenhouse to the field: demonstration of diminishing effects of mitigation of salinity in peppers inoculated with plant growth-promoting bacterium and humic acids. Appl Soil Ecol 119:327-338

Bakker PAHM, Berendsen RL, Doornbos RF, Wintermans PC, Pieterse CM (2013) The rhizosphere revisited: root microbiomics. Front Plant Sci 4:165

Bashan Y, de-Bashan LE, Prabhu SR, Hernandez JP (2014) Advances in plant growth-promoting bacterial inoculant technology: formulations and practical perspectives (1998-2013). Plant Soil 378:1-33

Bashan Y, Kloepper JW, de-Bashan LE, Nannipieri P (2016) A need for disclosure of the identity of microorganisms, constituents, and application methods when reporting tests with microbe-based or pesticide-based products. Biol Fertil Soils 52:283-284

Berg G, Zachow C, Müller H, Philipps J, Tilcher R (2013) Next generation bio-products sowing the seeds of success for sustainable agriculture. Agronomy 3:648-656

Berg G, Rybakova D, Grube M, Köberl M (2016) The plant microbiome explored: implications for experimental botany. J Exp Bot 67:9951002

Calvo P, Nelson L, Kloepper JW (2014) Agricultural uses of plant biostimulants. Plant Soil 383:3-41

De Bruin JL, Pedersen P, Conley SP, Gaska JM, Naeve SL, Kurle JE, Elmore RW, Giesler LJ, Abendroth LJ (2010) Probability of yield response to inoculants in fields with a history of soybean. Crop Sci $50: 265-272$

Denton MD, Coventry DR, Murphy PJ, Howieson JG, Bellotti WD (2002) Competition between inoculant and naturalised Rhizobium leguminosarum bv. trifolii for nodulation of annual clovers in alkaline soils. Aust J Agric Res 53:1019-1026

Karamanos R, Flore N, Harapiak J (2010) Re-visiting use of Penicillium bilaii with phosphorus fertilization of hard red spring wheat. Can J Plant Sci 90:265-277

Martínez-Viveros O, Jorquera MA, Crowley DE, Gajardo G, Mora ML (2010) Mechanisms and practical considerations involved in plant growth promotion by rhizobacteria. J Soil Sci Plant Nutr 10:293319

Nannipieri P, Penton CR, Purahong W, Scloter M, Van Elsas JD (2019) Recommendations for soil microbiome analyses. Biol Fertil Soils 55:765-766

Petsko GA (2010) When failure should be the option. BMC Biol 8:61

Posada LF, Alvarez JC, Hu CH, de-Bashan LE, Bashan Y (2016) Construction of probe of the plant growth-promoting bacteria Bacillus subtilis useful for fluorescence in situ hybridization. J Microbiol Meth128:125-129

Schöler A, Jacquiod S, Vestergaard G, Schulz S, Schloter M (2017) Analysis of soil microbial communities base on amplicon sequencing of marker genes. Biol Fertil Soils 53:485-489

Trejo A, de-Bashan LE, Hartmann A, Hernandez JP, Rothballer M, Schmid M, Bashan Y (2012) Recycling waste debris of immobilized microalgae and plant growth-promoting bacteria from wastewater treatment as a resource to improve fertility of eroded desert soil. Environ Exp Bot 75:65-73

Vestergaard G, Schulz S, Schöler A, Schloter M (2017) Making big data smart- how to use metagenomics to understand soil quality. Biol Fertil Soils 53:479-484

Publisher's note Springer Nature remains neutral with regard to jurisdictional claims in published maps and institutional affiliations. 